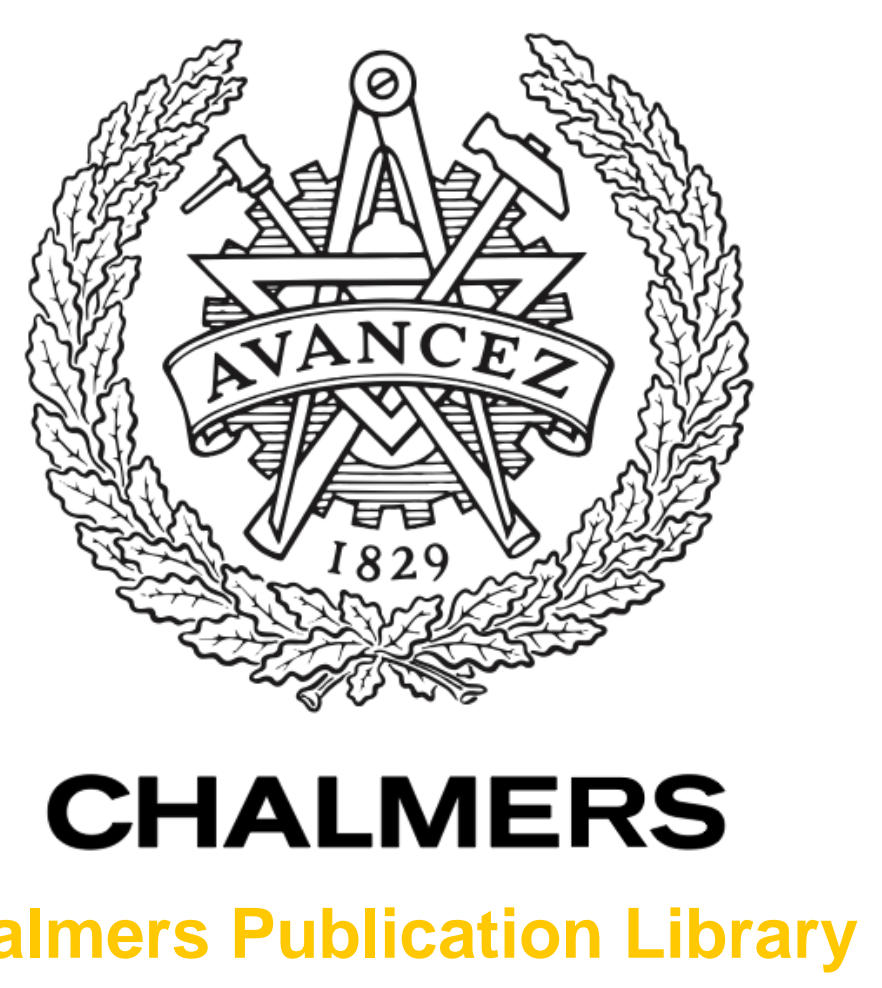

Chalmers Publication Library

\author{
Analysis and Design of RF Power and Data Link Using Amplitude Modulation of \\ Class-E for a Novel Bone Conduction Implant
}

This document has been downloaded from Chalmers Publication Library $(\mathrm{CPL})$. It is the author's version of a work that was accepted for publication in:

IEEE Transactions on Biomedical Engineering (ISSN: 0018-9294)

Citation for the published paper:

Taghavi, H. ; Håkansson, B. ; Reinfeldt, S. (2012) "Analysis and Design of RF Power and Data Link Using Amplitude Modulation of Class-E for a Novel Bone Conduction Implant". IEEE Transactions on Biomedical Engineering, vol. 59(11), pp. 3050-3059.

http://dx.doi.org/10.1109/TBME.2012.2213252

Downloaded from: http://publications.lib.chalmers.se/publication/164875

Notice: Changes introduced as a result of publishing processes such as copy-editing and formatting may not be reflected in this document. For a definitive version of this work, please refer to the published source. Please note that access to the published version might require a subscription. 


\title{
Analysis and Design of RF Power and Data Link Using Amplitude Modulation of Class-E for a Novel Bone Conduction Implant
}

\author{
Hamidreza Taghavi, Student Member, IEEE, Bo Håkansson, Sabine Reinfeldt
}

\begin{abstract}
This paper presents analysis and design of a radio frequency power and data link for a novel Bone Conduction Implant (BCI) system. Patients with conductive and mixed hearing loss and single-sided deafness can be rehabilitated by Bone Anchored Hearing Aids (BAHA). Whereas the conventional hearing aids transmit sound to the tympanic membrane via air conduction, the BAHA transmits sound via vibrations through the skull directly to the cochlea. It uses a titanium screw that penetrates the skin and needs life-long daily care; it may cause skin infection and redness. The $\mathrm{BCI}$ is developed as an alternative to the percutaneous BAHA since it leaves the skin intact. The BCI comprises an external audio processor with a transmitter coil and an implanted unit called the Bridging Bone Conductor with a receiver coil. Using Amplitude Modulation of the Class-E power amplifier that drives the inductive link, the sound signal is transmitted to the implant through the intact skin. It was found that the BCI can generate enough output force level for candidate patients. Maximum power output of the BCI was designed to occur at $5 \mathrm{~mm}$ skin thickness and the variability was within 1.5 dB for 1 to $8 \mathrm{~mm}$ skin thickness variations.
\end{abstract}

Index Terms-Bone Conduction Implant, RF power and data link, Class-E power amplifier, amplitude modulation, implantable hearing devices, Bone Anchored Hearing Aid, bone conduction devices, implantable transducer, low-power systems.

\section{INTRODUCTION}

A IR conduction hearing aids can sometimes not be used for patients who lack ear canal or have a middle ear disease. In these cases a conventional bone conduction (BC) hearing aid can be used with a vibrating bone conduction transducer. The sound is transmitted via vibrations through the skull bone directly to the cochlea. Conventional BC hearing aids can be uncomfortable to wear due to the static force pressing the transducer against the skin. Other drawbacks reported are reduced high frequency gain and feedback problems.

\section{A. Bone Anchored Hearing Aids}

To overcome the drawbacks of the conventional BC devices, a new model of $\mathrm{BC}$ hearing device named Bone Anchored Hearing Aid (BAHA) was introduced in the 1980s [1]. Fig. 1 shows the principal design of a generic BAHA, which has a percutaneous osseointegrated titanium screw anchored to the skull bone and a vibrating transducer connected to

Manuscript received April 30, 2012; revised June 29, 2012; accepted July 21, 2012. Date of publication —_- date of current version . The authors are affiliated with the Division of Signal Processing and Biomedical Engineering, Department of Signals and Systems, Chalmers University of Technology, Gothenburg, Sweden (email: taghavi@chalmers.se). Digital Object Identifier-

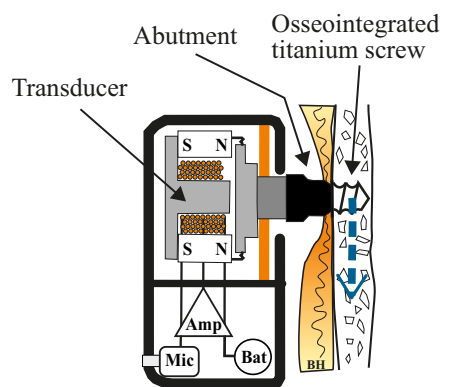

Fig. 1. Principal design of a generic percutaneous Bone Anchored Hearing Aid (BAHA) with a screw attachment to the skull bone. It comprises a microphone (Mic), battery (Bat) and pre and power amplifiers (Amp) that drives the bone conduction transducer.

the implant. The sound is picked up by a microphone and will then be amplified and effectively transmitted directly from the transducer to the skull bone. Thus, the external ear canal remains open and the middle ear is by-passed in the transmission of the sound.

The BAHA is suitable for patients who have a pure conductive deafness, mixed hearing loss (conductive and sensorineural), as well as for single sided deafness (SSD) and successful results have been reported [2], [3], [4]. However, the reports also present some drawbacks with the percutaneous BAHA. The skin penetrating implant requires a life-long commitment of everyday care. Other drawbacks are skin infection, skin redness, and risk for implant damage due to trauma [5], [6], [7], [8], [9]. Also the gain of the BAHA is limited to maintain the required gain headroom for stability.

\section{B. Bone Conduction Implant}

To solve the related drawbacks with the BAHA, a novel solution has been proposed, called a Bone Conduction Implant (BCI). The BCI is novel since the skin is kept intact and there is no skin penetrating implant with screw attachment. Instead, the transducer is implanted in the skull bone closer to the cochlea compared to using a BAHA. This implies that the BCI may have an increased sensitivity to bone conducted sound [10], [11], [12]. Moreover, the BCI has an improved gain headroom compared with the BAHA [13].

The principal design of the full-scale BCI system is shown in Fig. 2. The BCI comprises an external audio processor containing a microphone, a digital signal processor (DSP), analog signal processing parts and a tuned switching power amplifier that drives the transcutaneous inductive RF link to deliver 


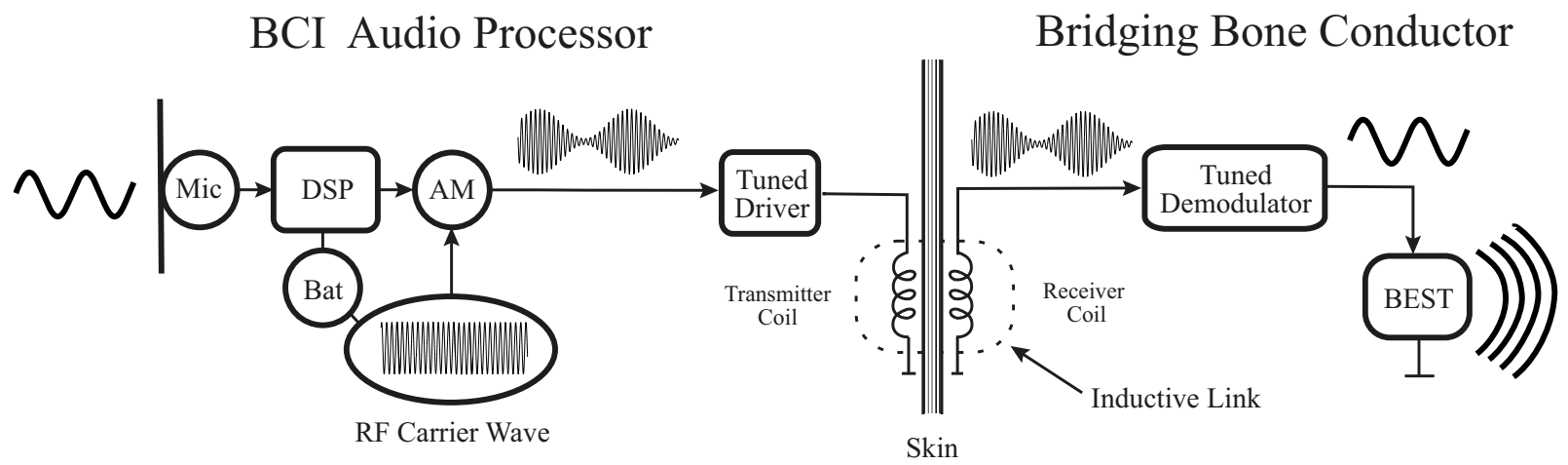

Fig. 2. Principal design of the full-scale BCI system. The sound signal is picked up by the microphone (Mic) and fed to the digital signal processor (DSP). It is then transmitted through the intact skin using amplitude modulation (AM) of the radio frequency (RF) carrier wave. The sound signal is extracted by the implanted tuned demodulating unit and then is fed to the BCI transducer, which uses the Balanced Electromagnetic Separation (BEST) principle.

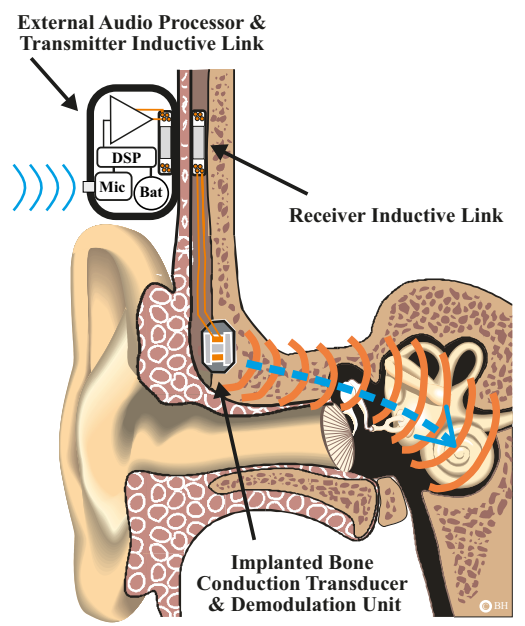

Fig. 3. The BCI system with an implanted and capsuled bone conduction transducer with a flat surface contact to the skull bone. It comprises also an inductive link and an externally worn audio processor. The vibrations are received to the cochlea through the temporal bone.

power and data to the transmitter. The implanted unit is called the Bridging Bone Conductor (BBC) and consists of a receiving tuned coil, demodulator and a bone conduction transducer. The BBC receives the power and amplitude modulated (AM) sound signal from transmitter coil transcutaneously via skin. Both transmitter and receiver coils are aligned by a permanent magnet retention system [11]. The anatomical positions of the implant and the externally worn audio processor are illustrated in Fig. 3. It is also shown how the vibrations are transmitted to the cochlea through the temporal bone.

Different methods can be performed to transmit power to the implant using inductive RF links [14], [15], [16] or optical methods [17]. Inductive RF links are more regularly used in transcutaneous power transmission. Some applications use an implanted rechargeable battery that supplies an internal processor, electrodes and electrode-control circuits, telemetry circuits or transducer. The battery can be recharged by using an inductive RF link [18] or optical techniques [17]. In several applications the data is also transmitted to the implant from the same inductive link using a modulation technique [19], [20]. The BBC consists of only passive components and therefore there is no requirement for regulated supply voltage for powering implanted components.

Taghavi et al. [21] has proposed an analog RF data and power link design for the BCI first generation, which could produce the highest possible output force level and a reasonable robust power transmission for different skin thicknesses. Even though the highest power transmission could be achieved, the robustness to the skin thickness variation was not satisfactory and several filters and AM circuitry had a current consumption that was too high. However, more efficient RF power amplifiers can be designed using switching technology such as Class-D [22], Class-E [23] and Class-DE [24]. In the next step it was therefore decided to investigate the use of the Class-E technology for the BCI in some more details.

\section{Aim of Study}

The aim of this study is to design and implement an efficient power and data transmission using drain amplitude modulation of a tuned Class-E power amplifier. The transmission robustness to the skin thickness variations will also be investigated.

\section{LOW-POWER RF POWER AND DATA LINK DESIGN}

Implantable biomedical devices are used in several medical conditions such as pacemakers for cardiac arrhythmia and cochlear implants for deafness. They need to be small and operate within low-power and low-voltage conditions to make them portable and easy to implant.

Fig. 4 presents the basic structure of an inductive RF tuned power and data link system for the BCI system. The AM power amplifier drives the tuned transmitter coil that sends energy and sound signal to the implanted unit inductively across the skin of the patient. On the receiver side, the RF signal passes through the envelope detector to extract the sound signal from the carrier wave. Simultaneously the power is transmitted to the implanted transducer. The BCI system is supplied by an external battery that provides energy for the entire external audio processor, RF link and the implanted circuits. In this paper, "coil spacing" is defined as the "skin thickness".

One very important design issue is that the RF link power transmission should be relatively insensitive to changes in a range of skin thicknesses of patients. There are several studies 


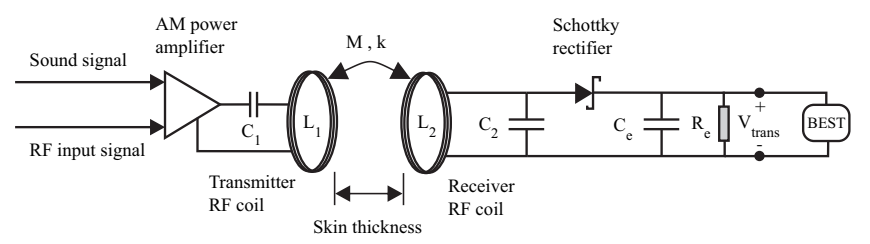

Fig. 4. Basic structure of an inductive RF tuned power and data link system for the BCI. The receiver includes an envelope detector, which generates $V_{\text {trans }}$ that drives the BEST transducer. The spacing (clearance) between capsuled coils is defined as the skin thickness.

on the theory and design of the RF links that recommend to tune the transmitter and receiver coils to make RF links less sensitive, not only to coil spacing, but also to lateral misalignment.

The most commonly approach, which is used for designing an inductive link, is the geometric method [25], [26], [27]. It has a transmitter coil that is larger in diameter than the implanted coil. In this method the gain is still sensitive to the changes in the coil spacing even though the coils can move laterally and even tilt somewhat with minor effect on the gain.

Another approach to design an inductive link is to operate near the critical coupling [15], [28]. In this method, higher overall efficiency can be achieved and the coil spacing tolerance will have small effect on the coupling gain. Using two tuned $\mathrm{RF}$ coils, the maximum coupling gain magnitude occurs at a value of geometric coupling coefficient that is called critical coupling $k_{\text {crit }}$. In $k_{\text {crit }}$, the gain function is nearly flat and therefore insensitive to changes in coupling coefficient (coil spacing). Maximum power transfer ratio in critical coupling is theoretically $50 \%$ but the efficiency will be less due to losses in the receiver coil. In both critical coupling and geometric methods the transmitter and receiver coils are tuned to the RF carrier signal frequency. Therefore, the bandwidth is poor and is not useful for wide band signal transmission. In [29] stagger tuning is used, which is based on pole placement where one resonance frequency is designed to be above the carrier frequency and one below. In this method, the gain of the link can be desensitized from the coupling variations. This design provides good efficiency and a large bandwidth. The most important parameters of the RF link, which should be considered when designing inductively coupled coils to transfer data and power, are the voltage gain, the power efficiency, the bandwidth, the RF power amplifier charactersitics and the implant loading effect.

\section{A. Theory of Linear Inductive Links}

A pair of magnetically coupled resonators is shown in Fig. 5 and represents a model of our RF data and power link with transmitter external coil $L_{1}$ on the left and receiver internal coil $L_{2}$ on the right. The mutual inductance between the transmitter and receiver coils is denoted by $M$. The geometric coupling coefficient $k$ exists due to the common flux linkage between two coils. For a uniform dielectric environment the coupling coefficient is in the range $0 \leq k \leq 1$. The mutual inductance directly relates to the coupling coefficient $k$ and (a)

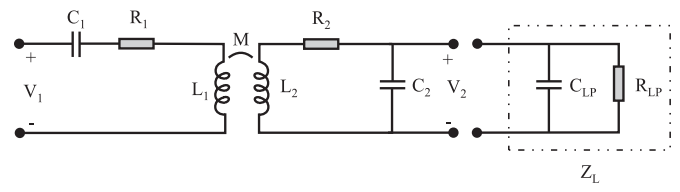

(b)

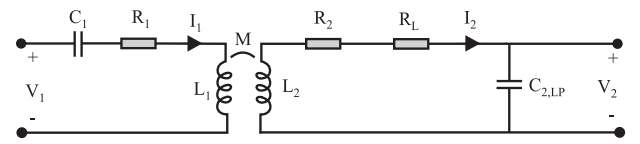

Fig. 5. Inductively coupled series-parallel resonators. (a) $Z_{L}$ consists of a parallel $R C$ network denoted as $C_{L P}$ and $R_{L P}$. (b) The $C_{L P}$ is absorbed into $C_{2}$, making $C_{2, L P}$, and the resistive part of $Z_{L}$ is transformed to its series equivalent denoted as $R_{L}$.

self inductances $L_{1}$ and $L_{2}$ by

$$
M=k \sqrt{L_{1} L_{2}} \text {. }
$$

There are methods to determine $k$ based on the geometry of the coils, see for example Hochmair [28] who computes $M$ with two circular coils facing each other. This method takes the coil spacing and lateral misalignment into account. To obtain $k$, the self-inductances are calculated using equations from [30]. Another method presented is by Soma et al. 1987 [31] who calculate the mutual inductance with perfect alignment, and with lateral and angular misalignments. Furthermore, in [25] an equation for calculating self-inductance is derived also taking the radius of coil's wire into account.

In Fig. 5 the inductively coupled series-parallel resonators are illustrated. The resistances $R_{1}$ and $R_{2}$ are implicit resistances due to coil losses in the transmitter and receiver coils while $C_{1}$ and $C_{2}$ are tuning capacitances used to create a resonance with $L_{1}$ and $L_{2}$, respectively. The receiver resonance circuit amplifies the induced voltage and helps to overcome the turn-on voltage of the Schottky rectifier in the envelope detector. A series tuned transmitter network needs lower swing voltage at its power amplifier output because the impedance of the $L_{1}$ and $C_{1}$ cancel at resonance. The envelope detector network together with the transducer in Fig. 4 that are in parallel with $C_{2}$ in parallel with $L_{2}$, have been replaced with an equivalent linear impedance $Z_{L}$ at the RF carrier frequency. $Z_{L}$ consists of a parallel $R C$ network, which is shown in Fig. 5(a). It is shown in Fig. 5(b) that the $C_{L P}$ is absorbed into $C_{2}$, creating $C_{2, L P}$, and the resistive part of $Z_{L}$ is transformed to its series equivalent, thus at resonance frequency, $R_{L}=\left(\omega L_{2}\right)^{2} / R_{L P}$.

The voltage transfer function of the the RF link according to Fig. 5(b) can be obtained by solving the following network equations:

$$
\left\{\begin{array}{l}
\left(R_{1}+j\left(\omega L_{1}-\frac{1}{\omega C_{1}}\right)\right) I_{1}+j \omega M I_{2}=V_{1} \\
j \omega M I_{1}+\left(R_{2}+R_{L}+j\left(\omega L_{2}-\frac{1}{\omega C_{2, L P}}\right)\right) I_{2}=0
\end{array}\right.
$$

where $\omega$ is the operating angular frequency and both transmitter and receiver coils are assumed to be tuned to the same resonance frequency $\omega_{0}$, so that

$$
\omega_{0}=\frac{1}{\sqrt{L_{1} C_{1}}}=\frac{1}{\sqrt{L_{2} C_{2, L P}}} .
$$


The voltage gain transfer function of the link obtained from equations 2, 3 and 4 can be expressed as:

$$
\frac{V_{2}}{V_{1}}=\frac{-\omega_{n r m}^{2} k \sqrt{\frac{L_{2}}{L_{1}}}}{\left[k^{2}+\frac{1}{Q_{1} Q_{2}^{\prime}}-\left(1-\omega_{n r m}^{2}\right)+j\left(1-\omega_{n r m}^{2}\right)\left(\frac{1}{Q_{1}}+\frac{1}{Q_{2}^{\prime}}\right)\right]}
$$

where $\omega_{n r m}=\omega / \omega_{0}$, and where $Q_{1}=\omega L_{1} / R_{1}$ and $Q_{2}=$ $\omega L_{2} / R_{2}$ are the unloaded quality factors of the transmitter and receiver coils, respectively. The loaded quality factor of the receiver is defined as $Q_{2}^{\prime}=\omega L_{2} /\left(R_{2}+R_{L}\right)$. The maximum voltage gain is achieved at the critical coupling coefficient $k_{\text {crit }}$, which is given by

$$
k_{c r i t}=\frac{1}{\sqrt{Q_{1} Q_{2}^{\prime}}} .
$$

For the condition of $\omega=\omega_{0}$ and $k=k_{\text {crit }}$, the receiver voltage is maximized and the peak gain is given by

$$
\left(\frac{V_{2}}{V_{1}}\right)_{M A X}=\frac{1}{2 k_{\text {crit }}} \sqrt{\frac{L_{2}}{L_{1}}}=\frac{\sqrt{Q_{1} Q_{2}^{\prime}}}{2} \sqrt{\frac{L_{2}}{L_{1}}} .
$$

Note that in equation 7 the maximum receiver voltage is independent of $k$.

\section{B. RF Link Power Efficiency}

The power efficiency of the RF link can be determined considering the fact that at $\omega=\omega_{0}$ and $k=k_{\text {crit }}$, both the transmitter and receiver impedances are purely resistive and therefore the reflected impedance from the receiver coil to the transmitter coil is purely resistive and added in series with the transmitter circuit. The power that is supplied by the source $V_{1}$ is dissipated in the load $R_{L}$ in two ways. Firstly, the reflected resistance $R_{r e f l}=\left(\omega^{2} M^{2}\right) /\left(R_{2}+R_{L}\right)$ and $R_{1}$ form a resistive divider and a fraction of the transmitter power is dissipated in the reflected resistance i.e. the receiver circuit. Secondly, the receiver resistance $R_{2}$ and the load $R_{L}$ form a resistive divider in the receiver circuit such that only a fraction of the power dissipated in the receiver is dissipated in the load $R_{L}$. The overall power transfer efficiency is the product of the energy efficiency of the two resistive dividers in the transmitter and the receiver circuits [16]. The dissipated power in the transmitter circuit is expressed as:

$$
P_{1}=\frac{V_{1}^{2}}{R_{1}+R_{\text {refl }}} .
$$

Therefore, the RF link efficiency is obtained from

$$
\eta_{\text {link }}=\frac{R_{\text {refl }}}{R_{1}+R_{\text {refl }}} \cdot \frac{R_{L}}{R_{2}+R_{L}},
$$

or expressed in quality factors

$$
\eta_{l i n k}=\frac{k^{2} Q_{1} Q_{2}^{\prime}}{1+k^{2} Q_{1} Q_{2}^{\prime}} \cdot \frac{Q_{2}}{Q_{2}+Q_{L}}
$$

where $Q_{L}=\omega L_{2} / R_{L}$.

\section{EXPERIMENTAL SYSTEM DESIGN}

The power amplifier, inductive link, envelope detector and the transducer contribute to the total efficiency of the BCI system. The overall efficiency of the BCI is the product of the RF power amplifier efficiency $\eta_{P A}$, the RF link efficiency $\eta_{\text {link }}$, the envelope detector efficiency $\eta_{e}$, and the transducer efficiency $\eta_{B E S T}$. The total efficiency can be expressed as

$$
\eta_{\text {total }}=\eta_{P A} \times \eta_{\text {link }} \times \eta_{e} \times \eta_{B E S T} .
$$

Equation 10 implies that for maximizing the link efficiency, the coupling coefficient $k$, unloaded quality factor of the transmitter coil $Q_{1}$, and the loaded quality factor of the receiver coil $Q_{2}^{\prime}$ need to be maximized and the load quality factor $Q_{L}$ must be minimized. On the other hand, $Q_{2}^{\prime}=\left(Q_{2} Q_{L} /\left(Q_{2}+Q_{L}\right)\right)$, which implies that a higher $Q_{L}$ results in a higher $\eta_{\text {link }}$. Thus, there is an optimal loading condition that is described in details in [15]. Coils have generally better $Q$ at higher frequencies, since the resistive skin effect losses is proportional to $\sqrt{\omega}$ while their reactance is proportional to $\omega$. However, the sensitivity to parasitics and tissue absorption increases with higher operating frequencies. Moreover, better timing precision is needed for the Class-E power amplifier driver. Considering these important issues, we have chosen to operate at $\omega_{0}=2 \pi \times f_{0}$ where $f_{0}=120 \mathrm{kHz}$. Single strand copper wires were used for the transmitter and receiver coils. The BCI design procedure starts with the inductive link and the resonator circuits based on the BEST transducer loading effect. The aim of this design is to transmit the maximum power to the implanted transducer and have high robustness of gain when the skin thickness changes over 1 to $10 \mathrm{~mm}$. Therefore, the inductive link was designed to operate around $k_{\text {crit }}$. Next step is to design the power amplifier to be reasonably efficient by optimizing the component values.

\section{A. Coupled Resonator Design}

In the BCI system, the sound is transferred to a transducer that converts the signal to bone vibrations. A new transducer principle was developed for implantation called the Balanced Electromagnetic Separation Transducer (BEST) [32], which has smaller size, lower distortion and higher efficiency than conventional bone conduction transducers. Therefore, the BEST electrical impedance $Z_{B E S T}$ characteristic is important when designing the inductive link. The impedance of the transducer that is used in the design is shown in Fig. 6. It should be noted that at the carrier frequency, the entire envelope detector together with the transducer reflect a parallel $R C$ network as illustrated in 5(a).

There are limitations on the physical size of the receiver and transmitter coils. The range of the coupling coefficients can be found considering the lateral misalignment and coil spacing. Equally-sized circular coils with the radius of $11 \mathrm{~mm}$ were chosen. The selected coil geometries can give a range of coupling coefficients $0.125<k<0.3$ within the required skin thickness range. It was shown in [33] that the skin flap thickness of Cochlear implant patients reaches $5 \mathrm{~mm}$ after 6 months. Therefore, it was assumed that $5 \mathrm{~mm}$ is the optimum skin thickness between the coils that has optimal coupling 

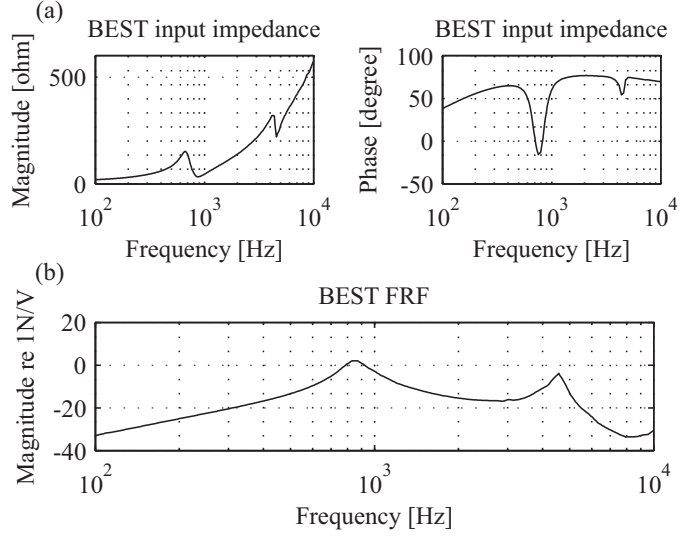

Fig. 6. (a) The electrical input impedance of the BEST presented as magnitude and phase, and (b) Frequency response function (FRF) of the BEST measured by a Skull simulator.

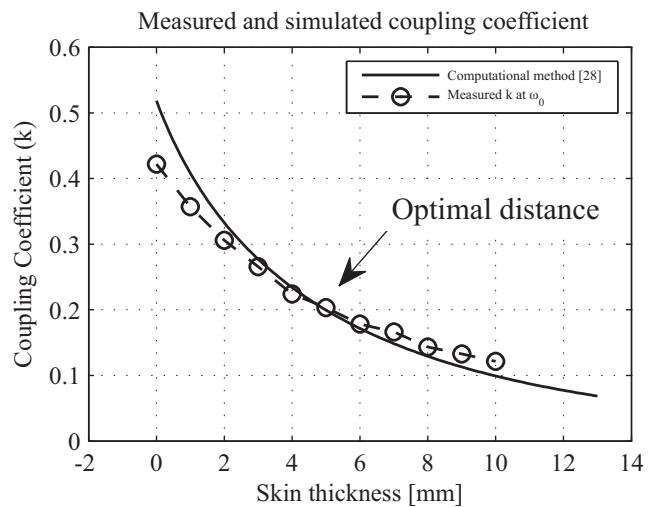

Fig. 7. Coupling coefficient $k$ as a function of skin thickness. The computational method in [28] is used. Dashed curve illustrates the measured $k$ between coils at carrier frequency $\omega_{0}$.

coefficient of $k=0.2$. Fig. 7 illustrates the measured $k$ and the computational method used in [28].

In a first design step, given the operating frequency and knowing the transducer loading effect together with the envelope detector, the receiver coil and receiver capacitance will be chosen. It is important to consider the reflected capacitance $C_{L P}$, which is absorbed into $C_{2}$, that contains the diode capacitance and the envelope detector filter capacitance. Also, the parasitic capacitance due to the self-resonance of the coil must be taken into account. This capacitance must be much smaller than $C_{2}$ to avoid shifts in tuning conditions.

In a second design step, the transmitter circuit parameters are determined by scaling the voltage gain when choosing the transmitter coil $L_{1}$ with respect to the receiver coil. Maximum possible voltage gain occurs at the critical coupling as given by equation 7 . Then $C_{1}$ is chosen in such a manner that it resonates with $L_{1}$ at $\omega_{0}$. Based on the fixed geometry and knowing the inductance values, the transmitter and receiver coils' number of turns can be determined using computational methods in [28]. In Fig. 8 the RF link voltage gain versus frequency and coupling coefficient according to equation 5 is plotted assuming the resistive part of the load impedance is $R_{L}=64 \Omega$. It can be seen that the maximum gain occurs

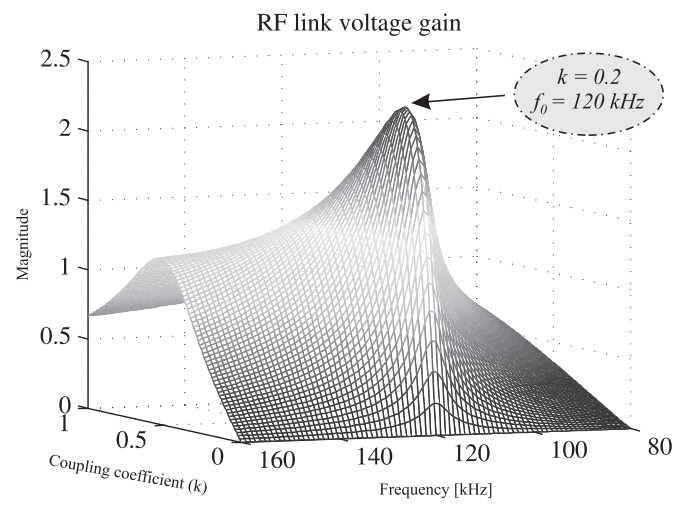

Fig. 8. RF link voltage gain vs. frequency and coupling coefficient.The maximum gain occurs at the resonance frequency of $120 \mathrm{kHz}$ and at optimal skin thickness of $5 \mathrm{~mm}$, which has a corresponding coupling coefficient of $k=k_{\text {crit }}=0.2$.

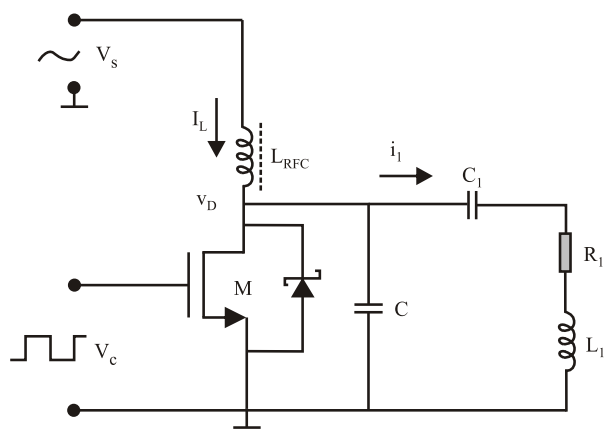

Fig. 9. AM Class-E tuned switching power amplifier connected to the transmitter circuit. $V_{c}$ is the carrier signal with the frequency of $f_{0}$ and $V_{s}$ is the processed sound signal that modulates the RF carrier signal. The Schottky diode is the drain-source (body) diode of the MOS transistor.

at the resonance frequency of $120 \mathrm{kHz}$ and at optimal skin thickness of $5 \mathrm{~mm}$ with a corresponding coupling coefficient of $k=k_{\text {crit }}=0.2$.

\section{B. Drain Amplitude Modulation of the Class-E RF Power Amplifier Design}

In Fig. 9 a tuned single-ended switching Class-E power amplifier topology comprising a big switching $\mathrm{N}$-channel transistor $M$, RF choke $L_{R F C}$, capacitors $C$ and $C_{1}$, resistor $R_{1}$, and coil $L_{1}$ is shown [23]. In a Class-E topology, the important voltage sources are carrier signal $V_{c}$, the source signal $V_{s}$ where the latter has a $d c$ level (approx. half the battery voltage) with the sound signal superimposed. Also there is one important node voltage $v_{D}$ that is drain potential. The MOS transistor $M$ is the active device that operates as a switch appropriately driven by a square wave at its gate to make it periodically turned on and off. The on resistance $R_{D S(o n)}$ of the transistor $M$ may be non-zero and the off resistance may be non-infinite. The shunt capacitance of the $d c$-feed choke $L_{R F C}$, the switch output capacitance, and wiring capacitance are all absorbed into $C$. The Schottky diode avoids the undershoots below zero at the node $v_{D}$. In a "nominal" Class-E operation, when $M$ is turned on, drain voltage $v_{D}$ is quickly pulled to ground and $L_{R F C}$ is fluxed up by the large voltage across it. $L_{R F C}$ is sufficiently large 
TABLE I

BCI RF LINK AND POWER DRIVER DESIGN SPECIFICATIONS

\begin{tabular}{ll}
\hline Parameter & Nominal Values \\
\hline Carrier frequency $f_{0}$ & $120 \mathrm{kHz}$ \\
Coil spacing (skin thickness) $d$ & $2 \mathrm{~mm}-10 \mathrm{~mm}$ \\
Class - E power NMOS & $R_{D S(\text { on }) \simeq 0.21 \Omega}$ \\
$L_{1}$ & for $V_{G S}=2.7 \mathrm{~V}$ \\
$L_{2}$ & $Q_{1} \simeq 24, r=11 \mathrm{~mm}$ \\
$L_{R F C}$ & $Q_{2} \simeq 19, r=11 \mathrm{~mm}$ \\
$Q_{L}$ & $\mathrm{RF}$ choke \\
$Q_{2}^{\prime}$ & 1.8 \\
$k_{\text {crit }}$ & 1.6 \\
Optimal coil spacing & 0.2 \\
Class - E supply voltage range & $5 \mathrm{~mm}$ \\
Optimal supply voltage & $0 \mathrm{~V}-1.3 \mathrm{~V}$ \\
\hline
\end{tabular}

to operate as a substantially constant current source $I_{L}$. When $M$ is turned off, capacitor $C$ is charged by the $d c$ current $I_{L} . v_{D}$ rises and stimulates the $R_{1} L_{1} C_{1}$ resonant transmitter circuit that converts the $d c$ energy to $a c$ energy at the switching frequency. The resonant circuit suppresses harmonics at $v_{D}$ to generate an approximately sinusoidal current $i_{1}$ in the transmitter. Capacitor $C$ will be charged and discharged by the difference between $d c$ and $a c$ currents. If the circuit parameters are precisely selected, at the switch "turn on" moment the return of $v_{D}$ to ground should be at zero voltage and zero slope. This is now known to be "nominal" Class-E operation [34]. The cycles will continue by charging and discharging $C$. The Class-E power amplifier shapes the voltage and current signals to prevent simultaneous high voltage and high current in the transistor that minimizes the power dissipation, especially during the switching transitions. A complete design procedure of the Class-E is described in [35].

In the Class-E power amplifier, the RF output amplitude is almost exactly a linear function of the $d c$ level of $V_{s}$, since conduction angles and wave shapes are not changed by the $d c$ supply voltage. Therefore, the RF output can be amplitude modulated by varying the $V_{s}$ in accordance with desired amplitude modulation envelope [36], [37]. Practically the AM Class-E power amplifier design includes four nonideal issues, which are very important in ultra-low-power systems. 1) First of all, the variations in the reflected load from receiver to transmitter, which adds in series to $R_{1}$, can change the damping characteristics of the transmitter circuit, which in turn causes $v_{D}$ to return to zero not at the switch "turn on" moment. This can dissipate $\frac{1}{2} f_{0} C v_{D}^{2}$ amount of energy when discharging currents in the switch $M$ and degrades the efficiency in the power amplifier. 2) The non-zero on resistance of the transistor $M, R_{D S(o n)}$ reduces the effective quality factor of the transmitter circuit and thus reduces the energy efficiency of the inductive link. 3) Gate capacitance of the transistor $M$ is periodically charged and discharged by the gate driver circuitry, which leads to energy losses in the switching. 4) In the drain amplitude modulation of the Class-E design, the supply voltage is substituted by a sound signal voltage source. Variations in the sound signal leads to variations in the output power to the load as the square of the supply

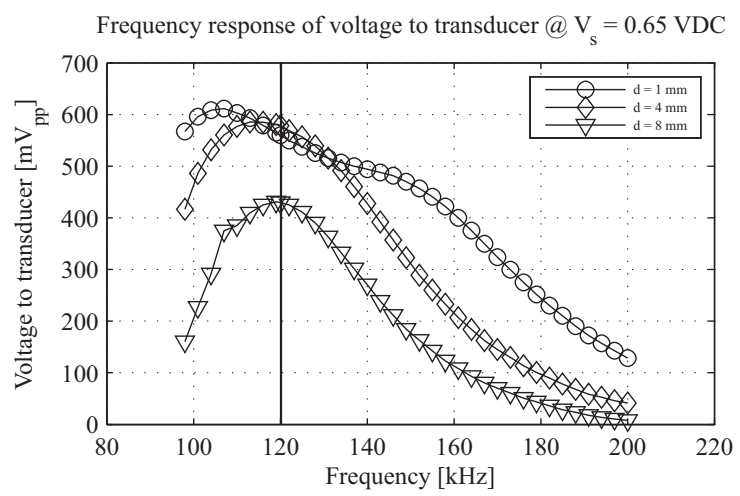

Fig. 10. Frequency response of the voltage to the transducer as the coil spacing is varied at $V_{s}=0.65 \mathrm{VDC}$. Maximum voltage to transducer occurs at $d=4 \mathrm{~mm}$ at the carrier frequency.

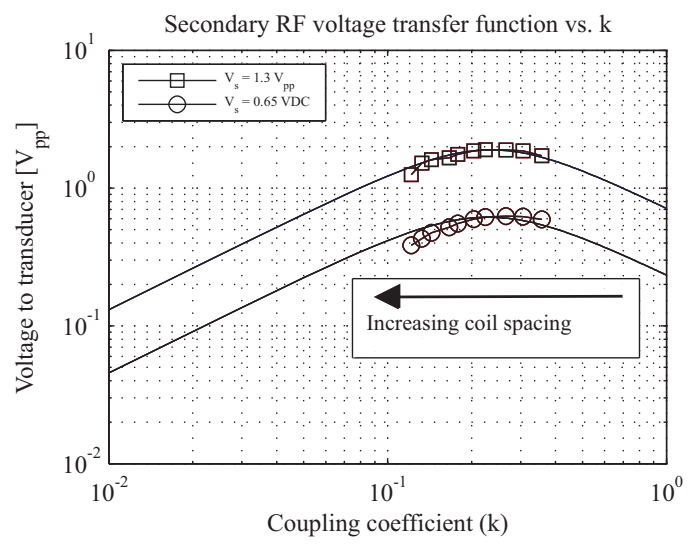

Fig. 11. Voltage transfer function of the voltage to transducer vs. coupling coefficient $k$. The peak voltage to the transducer occurs nearly at the critical coupling of $k_{\text {crit }} \approx 0.2$.

voltage. The load resistance was set at the correct value to give the desired output power at the median value of the $d c$ supply voltage (optimal supply voltage). Consequently the load resistance is kept unchanged when the $d c$ supply voltage changes. No parameters of the circuit will change, except for the transistor's output capacitance that will vary as the drain voltage changes. Another important issue, which has an effect on the reflected load from receiver to transmitter, is the input sound pressure level to the microphone that can change the amplitude of the RF signal. Accordingly, the impedance reflected from the envelope detector will change and hence the reflected resistance to the transmitter that changes the damping characteristic of the transmitter circuit.

In our design, nominal Class-E operation was designed and implemented for the BCI system. Tables I and II summarize the BCI system design specifications and the Class-E power amplifier drain efficiency simulated by HEPA-PLUS software [38], respectively.

Fig. 10 reveals the frequency transfer function of the voltage to transducer $V_{\text {trans }}$ as the coil spacing is varied. It can be seen that the maximum voltage to the transducer occurs at $4 \mathrm{~mm}$ coil spacing at the carrier frequency. At the carrier frequency, all three curves are nearly at their peaks. It is obvious that in this frequency, the voltage increases by moving 
TABLE II

CLASS-E DRAIN EFFICIENCY

\begin{tabular}{ll}
\hline Parameter & Simulated values \\
\hline$\eta_{\text {Class-E } @ 60 \mathrm{~dB} \mathrm{SPL}}$ & $86 \% @ d=4 \mathrm{~mm}$ \\
$\eta_{\text {Class-E }} @ 70 \mathrm{~dB}$ SPL & $87 \% @ d=4 \mathrm{~mm}$ \\
& $87 \% @ d=1 \mathrm{~mm}$ \\
$\eta_{\text {Class-E }} @ 90 \mathrm{~dB}$ SPL & $86 \% @ d=4 \mathrm{~mm}$ \\
& $64 \% @ d=8 \mathrm{~mm}$ \\
& $56 \% @ d=10 \mathrm{~mm}$ \\
\hline
\end{tabular}

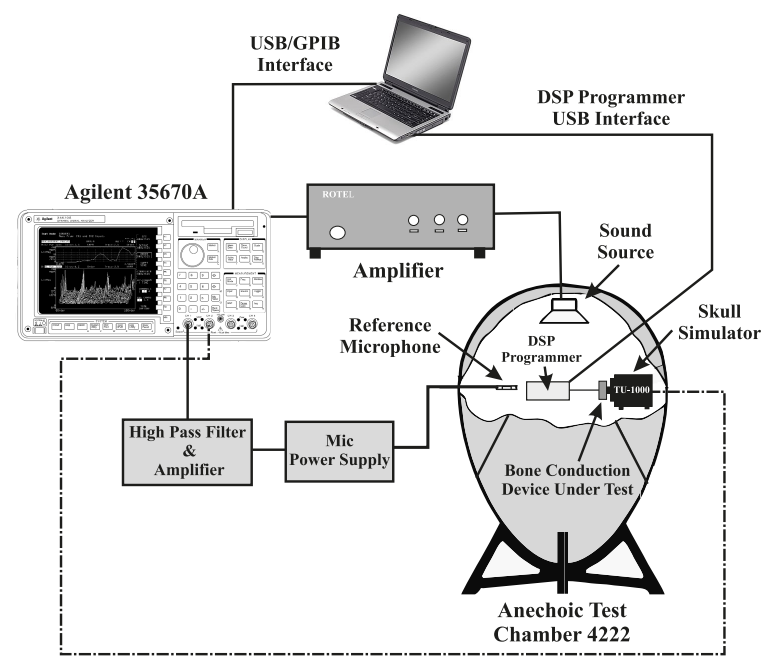

Fig. 12. Acoustical measurement set-up. The BCI system in the test is placed in the anechoic chamber and the transducer is attached to a Skull simulator. A signal analyzer generates the sound (sinus sweep 0.1-10 kHz) via an amplifier while the sound pressure is controlled by a reference microphone via a high pass filter \& amplifier. The analyzer measures the linear spectrum of the output force from the Skull simulator.

from maximum $k$ to $k_{c r i t}$ and then decreases towards the minimum $k$. This is the same behaviour that is expected by observing Fig. 8. It is shown in Fig. 11 that the peak voltage to the transducer occurs nearly at the critical coupling of $k_{\text {crit }} \approx 0.2$ that corresponds to the optimal coil spacing.

\section{Measurement Set-up And Methods}

In bone conduction devices, the input to the microphone is sound pressure and the output is force. Therefore, the performance test of the device was done by measuring the output force level (OFL) at different sound pressure levels (SPL). A Skull simulator was used to simulate the mechanical impedance of the skull bone and it generates a voltage proportional to the force applied at the connection point [39]. Measurements were performed in a Brüel \& Kjær anechoic test chamber type 4222 (Brüel \& Kjær Sound \& Vibration Measurement A/S, DK-2850 Nærum Denmark) see Fig. 12. A dynamic signal analyzer, Agilent 35670A (Agilent Technologies, Inc. Headquarters, Santa Clara CA, United States) was employed to generate and analyze signals. Automatic logarithmic swept sine in the frequency range of $100 \mathrm{~Hz}$ to $10 \mathrm{kHz}$ was used in all measurements. Linear spectrums of the output force were measured while the input sound pressure was kept constant at 60,70 and $90 \mathrm{~dB}$ SPLs with a reference Brüel \& Kjær microphone system type 2804. The impedances and

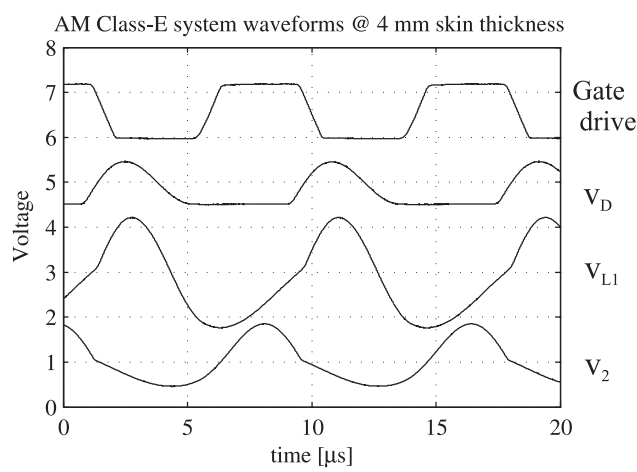

Fig. 13. BCI Class-E waveforms including the gate-driver signal, Class-E drain voltage $v_{D}$, voltage to the transmitter coil $v_{L 1}$ and receiver voltage $v_{2}$ for a $4 \mathrm{~mm}$ coil spacing condition.

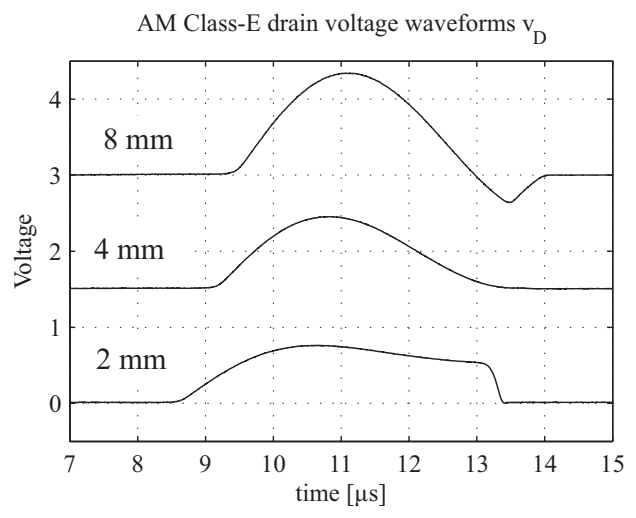

Fig. 14. Class-E drain voltage $v_{D}$ in three cases are shown. The optimal Class-E drain voltage occurs at the $4 \mathrm{~mm}$ coil spacing. Furthermore, it is obvious that the behaviour of the protection diode of the NMOS power transistor occurs in the $d=8 \mathrm{~mm}$ condition where the drain voltage had dropped below ground.

coupling coefficient of the RF link were measured in different set-ups using Network/Spectrum Analyzer HP 4195A.

\section{Measured Performance and Results}

The BCI system was implemented accordingly to the design procedure for both audio processor and the receiver circuit. All tests had a supply voltage of $1.3 \mathrm{VDC}$. The transmitter coil $L_{1}$ had a quality factor of 24. However, the NMOS switch in the Class-E adds another resistance to the transmitter coil, which reduces the quality factor to

$$
Q_{1, \text { effective }}=\frac{\omega L_{1}}{R_{1}+1.365 R_{D S(o n)}} .
$$

Therefore, the $k_{c r i t}$ will increase according to equation 6 and hence the maximum voltage gain occurs at a slightly closer distance between coils. Furthermore, the dissipated power of $R_{D S(\text { on })} I_{L}^{2}$ will be larger, which reduces the efficiency.

In Fig. 13 the designed nominal Class-E waveforms of the BCI system are shown. Fig. 14 illustrates the Class-E drain voltage $v_{D}$ over the changes in the coil spacing. In Fig. 15 the input sound of $1 \mathrm{kHz}$ with $90 \mathrm{~dB}$ SPL to the microphone and resulting amplitude modulated RF signal to the transmitter coil in the $4 \mathrm{~mm}$ coil spacing are shown. In Fig. 16 the BCI 


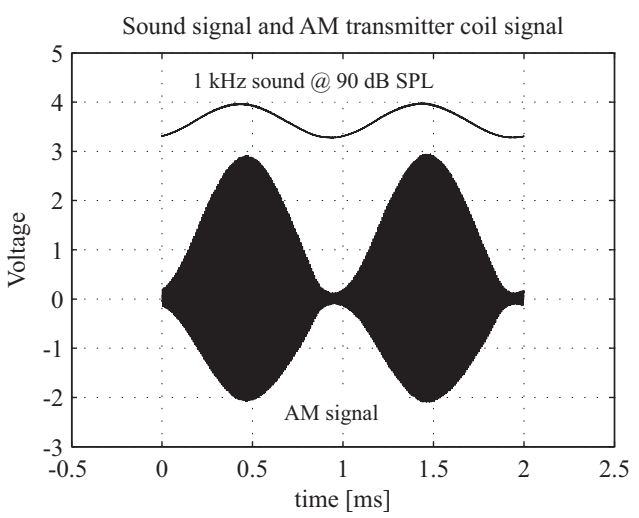

Fig. 15. Amplitude modulated RF signal of the transmitter coil from a 1 $\mathrm{kHz}$ sound signal of $90 \mathrm{~dB}$ SPL to the microphone. The coil spacing is 4 $\mathrm{mm}$.

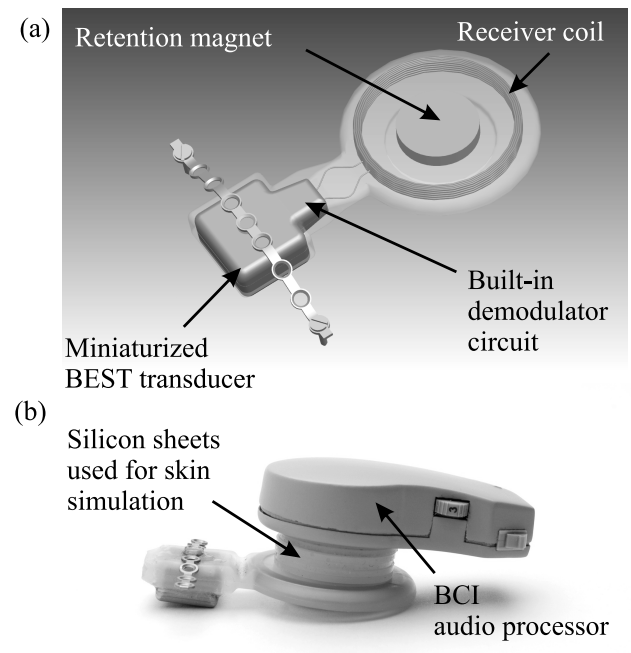

Fig. 16. (a) Bridging Bone Conductor including receiver coil, permanent retention magnet to keep the audio processor and implant attached to the patient's skin, built-in demodulator and receiver tuning circuit, bar construction and orthopaedic screws, and the miniaturized BEST transducer. (b) BCI audio processor containing the battery compartment, volume control, DSP, AM transmitter circuitry, transmitter coil and a retention magnet. Silicon sheets were used for skin thickness simulation.

system audio processor and the implanted unit called the BBC with detailed components are presented.

The acoustical performance of the BCI system measured using a Skull simulator is shown in Fig. 17. OFLs of the BCI were measured at 60,70 and $90 \mathrm{~dB}$ SPLs where the 60 and $70 \mathrm{~dB}$ SPL curves reveal that the device is linear as $10 \mathrm{~dB}$ increase in the input sound pressure level results in $10 \mathrm{~dB}$ increase in the output force level. The most important curve is the maximum power output (MPO) when the device is saturated and limited by the battery voltage capacity at the output. The MPO curves were also measured for different coil spacing (skin thickness). It can be seen that the MPOs will change $1.5 \mathrm{~dB}$ over the skin thickness range of 1 to $8 \mathrm{~mm}$. It is illustrated that the new BEST design has the high frequency boost that can be beneficial for speech understanding [11], [40]. Furthermore, it is illustrated in Fig. 17(b) that the input sound pressure to the microphone was fairly constant and (a) OFL linear spectrum of the $\mathrm{BCI}$

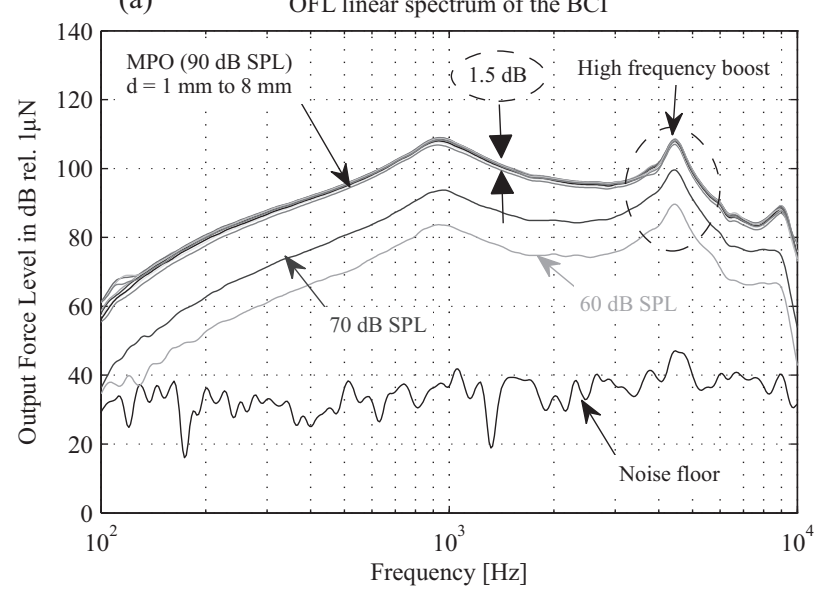

(b)

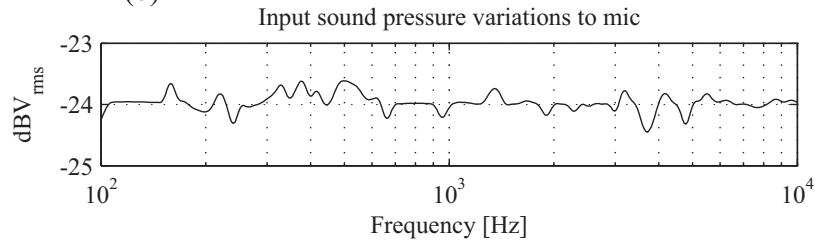

Fig. 17. (a) Output force levels (OFL) measured at 60, 70 and $90 \mathrm{~dB}$ SPL. Maximum power output (MPO) curves were measured where the coil spacing was varied from 1 to $8 \mathrm{~mm}$. Highest MPO occurs in the $4 \mathrm{~mm}$ skin thickness. The noise floor curve shows that the measurements were done with a high signal to noise ratio (SNR). Between 4 and $5 \mathrm{kHz}$ the BEST transducer has a second resonance frequency to boost the high frequency response. (b) The sound pressure was kept fairly constant during the entire frequency sweep $( \pm 0.5 \mathrm{~dB})$.

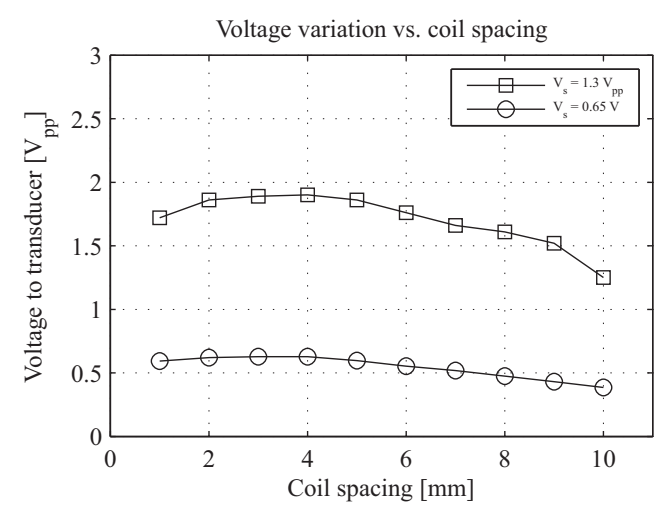

Fig. 18. Variation in the peak to peak voltage to the transducer $\left(V_{\text {trans }}\right)$ for two different sound voltage levels. In the saturation condition $V_{s}=1.3 V_{p p}$, the voltage variation is less than $16 \%$ for coil spacing range of 2 to $8 \mathrm{~mm}$.

changing only $\pm 0.5 \mathrm{~dB}$ in the entire frequency sweep.

The variation of the peak to peak voltage to the transducer over a range of coil spacings is shown in Fig. 18. Two levels of sound signal $V_{s}=0.65 V D C$ (no signal), which is the optimal supply voltage in the Class-E design, and $V_{s}=1.3 V_{p p}$, which is the saturation condition of the device, were measured. It is obvious that maximum voltages to transducer occur around $4 \mathrm{~mm}$ skin thickness in both conditions. Moreover, in the saturation condition, the difference between the minimum and maximum voltage is less than $16 \%$. 
(a)

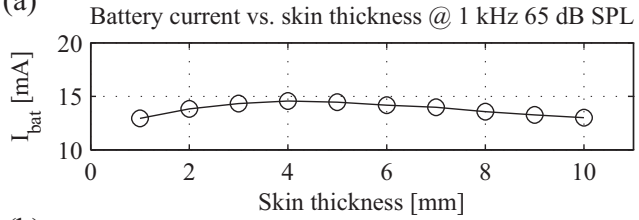

(b)

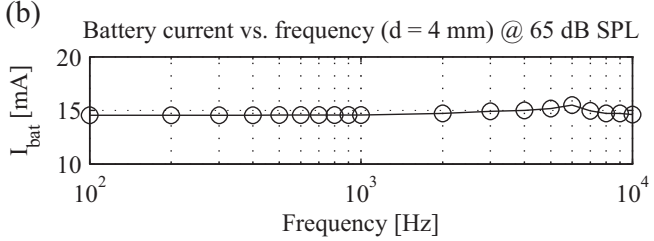

Fig. 19. (a) The BCI battery current consumption with the input signal of $1 \mathrm{kHz}$ at $65 \mathrm{~dB}$ SPL when the skin thickness varied from 1 to $10 \mathrm{~mm}$. The highest current consumption is at $d=4 \mathrm{~mm}$. (b) The BCI current consumption in the $4 \mathrm{~mm}$ skin thickness in frequency range of $100 \mathrm{~Hz}$ to $10 \mathrm{kHz}$ with $65 \mathrm{~dB}$ input sound pressure level. The current consumption increases slightly around $6 \mathrm{kHz}$.

\section{DISCUSSION}

By a more detailed look at the MPO curves in Fig. 17, it was found that that the maximum output of the BCI in this design occurs at $4 \mathrm{~mm}$ skin thickness. The design was done to have the maximum output at $5 \mathrm{~mm}$ skin thickness, but the contributions of the NMOS transistor on resistance $R_{D S(\text { on })}$ and the equivalent series resistances of $C$ and $C_{1}$ will reduce the quality factor of the transmitter circuit that results in changing the transmitter tuning condition. However, this influence is minor and it can be seen that the output force levels are fairly robust for skin thickness changes over 1 to $8 \mathrm{~mm}$ variations. The maximum MPO at the transducer resonance frequency in the $4 \mathrm{~mm}$ skin thickness was found to be $109 \mathrm{~dB}$ relative to $1 \mu \mathrm{N}$, i.e. $4 \mathrm{~dB}$ higher than the previous BCI system design [21], which may be beneficial for patients. Maximum MPO of a conventional BAHA device at transducer resonance frequency is $116 \mathrm{~dB}$ relative to $1 \mu \mathrm{N}$, but the $\mathrm{BCI}$ transducer is also positioned closer to the cochlea, which may increase the bone conducted sound by 10-15 dB [10], [11]. One important efficiency measure of the device is the battery current consumption measured according to [41]. Fig. 19 (a) illustrates the measured battery drain current of the BCI system based on the ANSI/ASA S3.22-2009 American National Standard for specification of hearing aid characteristics (revision of ANSIS 3.22-2003) [41]. The battery current was measured with an input sinusoidal of $1 \mathrm{kHz}$ at $65 \mathrm{~dB}$ SPL when the skin thickness was changed from 1 to $10 \mathrm{~mm}$. Maximum current consumption is at $4 \mathrm{~mm}$ skin thickness $(d=4 \mathrm{~mm})$. Fig. 19 (b) shows the battery current consumption in this skin thickness in the frequency range of $100 \mathrm{~Hz}$ to $10 \mathrm{kHz}$ at $65 \mathrm{~dB}$ SPL. Table III summarizes the current consumption of each part of the BCI system.

\section{CONCLUSion AND Future Work}

A novel bone conduction device called the Bone Conduction Implant (BCI) has been designed and implemented as an alternative to the percutaneous bone anchored hearing aid. The BCI keeps the skin intact by using an inductive link to transmit
TABLE III

BCI SYSTEM CURRENT CONSUMPTIONS

\begin{tabular}{lc}
\hline The BCI part & Current consumption (mA) \\
\hline DSP and microphone amplifier & 1 \\
Filters & 2.5 \\
Gate driver & 0.5 \\
Class - E loaded with receiver in the & 11 \\
4 mm skin thickness @ 65 dB SPL & \\
\hline
\end{tabular}

power and data to the implanted transducer wirelessly. It was concluded that the $\mathrm{BCI}$ with a properly designed ClassE power amplifier

- can generate high enough output force level for candidate patients as compared to conventional BAHA devices,

- is fairly insensitive to skin thickness variations for power transmission to the implant and

- has acceptable current consumption especially in relation to maximum power output.

It was found that this design has high current consumption in low SPLs as the control and filter circuits of the device have high quiescent currents. In future work, the filtering and gate-driver circuitry as well as the power amplifier will be designed in an application specific integrated circuit (ASIC). Since the Class-E power amplifier behaves more like a current source, other amplitude modulation topologies will be tested using Class-D or Class-DE, which operate more like voltage sources.

\section{ACKNOWLEDGMENT}

The authors would like to thank Dr. Nathan O. Sokal for making invaluable suggestions for improvement of the design. Also, the authors thank VINNOVA, the Swedish Governmental Agency for Innovations Systems that is supporting the project.

\section{REFERENCES}

[1] B. Håkansson, A. Tjellström, U. Rosenhall, and P. Carlsson, "The bone-anchored hearing aid: principal design and a psychoacoustical evaluation," Acta oto-laryngologica, vol. 100, no. 3-4, pp. 229-239, 1985.

[2] A. Snik, E. Mylanus, and C. Cremers, "The bone-anchored hearing aid compared with conventional hearing aids. Audiologic results and the patients' opinions." Otolaryngologic Clinics of North America, vol. 28, no. 1, p. 73, 1995.

[3] A. Tjellström, B. Håkansson, G. Granström et al., "Bone-anchored hearing aids: current status in adults and children." Otolaryngologic Clinics of North America, vol. 34, no. 2, p. 337, 2001.

[4] A. Snik, E. Mylanus, D. Proops, J. Wolfaardt, W. Hodgetts, T. Somers, J. Niparko, J. Wazen, O. Sterkers, C. Cremers et al., "Consensus statements on the baha system: where do we stand at present?" The Annals of otology, rhinology \& laryngology. Supplement, vol. 195, p. 2, 2005.

[5] A. Tjellström and G. Granström, "Long-term follow-up with the boneanchored hearing aid: a review of the first 100 patients between 1977 and 1985." Ear, nose, \& throat journal, vol. 73, no. 2, p. 112, 1994.

[6] E. Myers, R. Reyes, A. Tjellström, and G. Granström, "Evaluation of implant losses and skin reactions around extraoral bone-anchored implants: a 0-to 8-year follow-up," Otolaryngology-Head and Neck Surgery, vol. 122, no. 2, p. 272, 2000.

[7] R. Battista and P. Littlefield, "Revision baha surgery." Otolaryngologic Clinics of North America, vol. 39, no. 4, p. 801, 2006.

[8] M. Shirazi, S. Marzo, and J. Leonetti, "Perioperative complications with the bone-anchored hearing aid," Otolaryngology-Head and Neck Surgery, vol. 134, no. 2, p. 236, 2006. 
[9] J. Wazen, B. Wycherly, and J. Daugherty, "Complications of boneanchored hearing devices," Implantable Bone Conduction Hearing Aids, vol. 71, pp. 63-72, 2011.

[10] B. Håkansson, M. Eeg-Olofsson, S. Reinfeldt, S. Stenfelt, and G. Granström, "Percutaneous versus transcutaneous bone conduction implant system: a feasibility study on a cadaver head," Otology \& Neurotology, vol. 29, no. 8, p. 1132, 2008.

[11] B. Håkansson, S. Reinfeldt, M. Eeg-Olofsson, P. Östli, H. Taghavi, J. Adler, J. Gabrielsson, S. Stenfelt, and G. Granström, "A novel bone conduction implant BCI: engineering aspects and pre-clinical studies," International journal of audiology, vol. 49, no. 3, pp. 203-215, 2010.

[12] B. Håkansson, "The future of bone conduction hearing devices," Implantable Bone Conduction Hearing Aids, vol. 71, pp. 140-152, 2011.

[13] H. Taghavi, B. Håkansson, S. Reinfeldt, M. Eeg-Olofsson, and S. Akhshijan, "Feedback analysis in percutaneous bone-conduction device and bone-conduction implant on a dry cranium," Otology \& Neurotology, vol. 33, no. 3, p. 413, 2012.

[14] G. Wang, W. Liu, M. Sivaprakasam, and G. Kendir, "Design and analysis of an adaptive transcutaneous power telemetry for biomedical implants," Circuits and Systems I: Regular Papers, IEEE Transactions on, vol. 52, no. 10 , pp. 2109-2117, 2005.

[15] M. Baker and R. Sarpeshkar, "Feedback analysis and design of RF power links for low-power bionic systems," Biomedical Circuits and Systems, IEEE Transactions on, vol. 1, no. 1, pp. 28-38, 2007.

[16] R. Sarpeshkar, Ultra low power bioelectronics: fundamentals, biomedical applications, and bio-inspired systems. Cambridge Univ Pr, 2010.

[17] K. Goto, T. Nakagawa, O. Nakamura, and S. Kawata, "An implantable power supply with an optically rechargeable lithium battery," Biomedical Engineering, IEEE Transactions on, vol. 48, no. 7, pp. 830-833, 2001

[18] H. Lim, Y. Yoon, C. Lee, I. Park, B. Song, and J. Cho, "Implementation of a transcutaneous charger for fully implantable middle ear hearing device," in Engineering in Medicine and Biology Society, 2005. IEEEEMBS 2005. 27th Annual International Conference of the. IEEE, 2006, pp. 6813-6816.

[19] P. Troyk and M. Schwan, "Closed-loop class E transcutaneous power and data link for microimplants," Biomedical Engineering, IEEE Transactions on, vol. 39, no. 6, pp. 589-599, 1992.

[20] K. Iniewski, VLSI circuits for biomedical applications. Artech House Publishers, 2008.

[21] H. Taghavi, B. Håkansson, and S. Reinfeldt, "A novel bone conduction implant-analog radio frequency data and power link design," in Biomedical Engineering/765: Telehealth/766: Assistive Technologies. ACTA Press, 2012.

[22] S. El-Hamamsy, "Design of high-efficiency RF class-D power amplifier," Power Electronics, IEEE Transactions on, vol. 9, no. 3, pp. 297-308, 1994.

[23] N. Sokal and A. Sokal, "Class E a new class of high-efficiency tuned single-ended switching power amplifiers," Solid-State Circuits, IEEE Journal of, vol. 10, no. 3, pp. 168-176, 1975.

[24] H. Koizumi, T. Suetsugu, M. Fujii, K. Shinoda, S. Mori, and K. Iked, "Class DE high-efficiency tuned power amplifier," Circuits and Systems I: Fundamental Theory and Applications, IEEE Transactions on, vol. 43, no. 1, pp. 51-60, 1996

[25] C. Zierhofer and E. Hochmair, "Geometric approach for coupling enhancement of magnetically coupled coils," Biomedical Engineering, IEEE Transactions on, vol. 43, no. 7, pp. 708-714, 1996.

[26] N. Donaldson and T. Perkins, "Analysis of resonant coupled coils in the design of radio frequency transcutaneous links," Medical and Biological Engineering and Computing, vol. 21, no. 5, pp. 612-627, 1983.

[27] W. Ko, S. Liang, and C. Fung, "Design of radio-frequency powered coils for implant instruments," Medical and Biological Engineering and Computing, vol. 15, no. 6, pp. 634-640, 1977.

[28] E. Hochmair, "System optimization for improved accuracy in transcutaneous signal and power transmission," Biomedical Engineering, IEEE Transactions on, no. 2, pp. 177-186, 1984.

[29] D. Galbraith, M. Soma, and R. White, "A wide-band efficient inductive transdennal power and data link with coupling insensitive gain," Biomedical Engineering, IEEE Transactions on, no. 4, pp. 265-275, 1987.

[30] F. Terman, Radio engineers' handbook. McGraw-Hill New York, 1943, vol. 2.

[31] M. Soma, D. Galbraith, and R. White, "Radio-frequency coils in implantable devices: misalignment analysis and design procedure," Biomedical Engineering, IEEE Transactions on, no. 4, pp. 276-282, 1987.

[32] B. Håkansson, "The balanced electromagnetic separation transducer: A new bone conduction transducer," The Journal of the Acoustical Society of America, vol. 113, p. 818, 2003 .
[33] C. Raine, C. Lee, D. Strachan, C. Totten, and S. Khan, "Skin flap thickness in cochlear implant patients-a prospective study," Cochlear Implants International, vol. 8, no. 3, pp. 148-157, 2007.

[34] N. Sokal, "Direct contact to Dr. Sokal about correction to "optimum" class-E switch-voltage waveform."

[35] N. O. Sokal, "Class-E high-efficiency RF/microwave power amplifiers: Principles of operation, design procedures, and experimental verification," Analog Circuit Design, pp. 269-301, 2003.

[36] F. Raab, "Idealized operation of the class E tuned power amplifier," Circuits and Systems, IEEE Transactions on, vol. 24, no. 12, pp. 725735, 1977.

[37] M. Kazimierczuk, "Collector amplitude modulation of the class E tuned power amplifier," Circuits and Systems, IEEE Transactions on, vol. 31, no. 6, pp. 543-549, 1984.

[38] "HEPA-PLUS computer program for design, simulation and optimization of high-efficiency RF/microwave power amplifiers, Copyrighted 1990 Design Automation Inc."

[39] B. Håkansson and P. Carlsson, "Skull simulator for direct bone conduction hearing devices," Scandinavian audiology, vol. 18, no. 2, pp. 91-98, 1989.

[40] B. Hakansson, "Bone conduction transducer with improved high frequency response," Apr. 5 2012, US Patent 20,120,083,860.

[41] ANSI, "ANSI s3. 22-2003, specification of hearing aid characteristics," 2003.

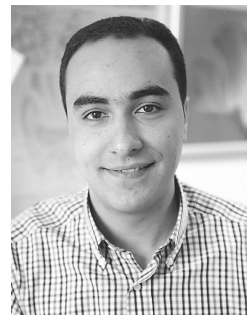

Hamidreza Taghavi received the M.Sc. degree in biomedical engineering in 2009 from Chalmers University of Technology, Gothenburg, Sweden where he is currently pursuing the Ph.D. degree on the novel Bone Conduction Implant system. His research interests include hearing implants, bone anchored hearing aids, bone conduction hearing implants, low-power integrated circuit design for implantable medical devices, biomedical instrumentations, and integrated radio frequency circuits.

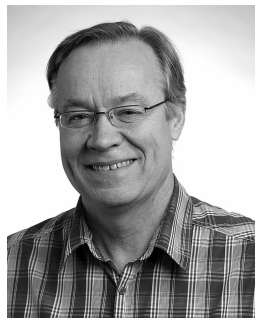

Bo Håkansson received his M.Sc. degree in 1977 in Electrical Engineering from Chalmers University of Technology, Gothenburg, Sweden. He received his $\mathrm{Ph} . \mathrm{D}$. degree in 1984 by defending the thesis with title "The Bone Anchored Hearing Aid- Engineering Aspects" at the department of Applied Electronics, Chalmers University of Technology, Gothenburg, Sweden. Currently he is Professor in Biomedical Engineering and head of the Biomedical signals and systems research group at department of Signals and Systems, Chalmers University of Technology, Gothenburg, Sweden. The research areas are focused on different applications of bone conduction hearing. Specifically his research is directed to implantable hearing devices, audiometric transducers, communication systems and conventional hearing aids all based on bone conduction. This research has emanated in six Ph.D. theses and some 60 scientific publications and 19 patents. He is also giving courses on Bachelor and Master levels regarding Microelectronic circuits and Biomedical instrumentation.

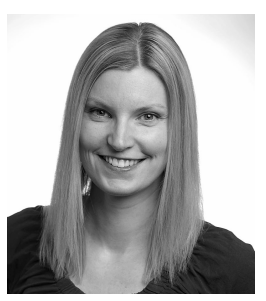

Sabine Reinfeldt received her M.Sc. degree in 2003 in Engineering Physics with focus on Biomedical Engineering from Chalmers University of Technology, Gothenburg, Sweden. She received her Ph.D. degree in 2009 by defending the thesis with title "Bone Conduction Hearing in Human Communication - Sensitivity, Transmission, and Applications" at the department of Signals and Systems, Chalmers University of Technology, Gothenburg, Sweden. Currently she has a Postdoc position at the same department, where she performs research about a new Bone Conduction Implant System and teaches Biomedical Instrumentation to students in the Master Program of Biomedical Engineering. Her research interests include bone conduction hearing, bone conduction devices, hearing implants, occlusion effect, bone conduction transmission and sensitivity, human communication, and one's own voice. 